\title{
OrclassWeb: A Tool Based on the Classification Methodology ORCLASS from Verbal Decision Analysis Framework
}

\author{
Thais Cristina Sampaio Machado, Plácido Rogerio Pinheiro, and Isabelle Tamanini \\ Graduate Program in Applied Informatics, University of Fortaleza (UNIFOR), Avenue Washington Soares 1321, Bl J Sl 30 , \\ 60811-905 Fortaleza, CE, Brazil \\ Correspondence should be addressed to Plácido Rogerio Pinheiro; placidrp@uol.com.br
}

Received 11 April 2014; Revised 10 July 2014; Accepted 11 July 2014; Published 24 July 2014

Academic Editor: Ker-Wei Yu

Copyright (C) 2014 Thais Cristina Sampaio Machado et al. This is an open access article distributed under the Creative Commons Attribution License, which permits unrestricted use, distribution, and reproduction in any medium, provided the original work is properly cited.

\begin{abstract}
The decision making is present in every activity of the human world, either in simple day-by-day problems or in complex situations inside of an organization. Sometimes emotions and reasons become hard to separate; therefore decision support methods were created to help decision makers to make complex decisions, and Decision Support Systems (DSS) were created to aid the application of such methods. The paper presents the development of a new tool, which reproduces the procedure to apply the Verbal Decision Analysis (VDA) methodology ORCLASS. The tool, called OrclassWeb, is software that supports the process of the mentioned DSS method and the paper provides proof of concepts, that which presents its reliability with ORCLASS.
\end{abstract}

\section{Introduction}

Present in every human activity, the decision may be defined because of a process of choice, given an identified problem or when the decision maker faces an opportunity of creation, optimization, or improvement in an environment. Aiming to solve complex problems, difficultly to be resolved by human process capacity in a qualitative way, Decision Support Systems (DSS) are methodologies that help a decision maker to rank or classify multiattribute alternatives, according to [1].

On the context of Multiple Criteria Decision Analysis (MCDA) classification approaches, we have several methods to be considered. The MCDA classification approaches can be subdivided into two types [2], described as follows.

(i) Parameter-Based Methods. The approach works with constructing a formal model of the decision maker's behavior based on some parameters, and this approach can be subdivided into utility function-based and outranking relationbased models [2]. These methods can also be categorized further into direct and indirect. In this area, methods such as ELECTRE TRI, PROAFTN, and UTADIS can be cited, among various others. The work presented in [3] proposes a new classification method based on ECLECTRE IV and on a customized genetic algorithm, which are employed in order to select the prototypes (alternatives that serve as class representatives related to a given problem) and calibrate the control parameters automatically.

(ii) Parameter-Free Methods. This approach is characterized for containing decision rule methods. It assumes that the Decision Maker's behavior is too complex to be mapped as parameters, and thus, proposes that it can be described in a symbolic or judgmental form by means of decision rules [2]. The idea was originated based on machine learning techniques, such as expert systems. Among the approaches that belong to the model, we have the Verbal Decision Analysis (VDA) methods and methods based on fuzzy logic. The VDA framework is structured on the acknowledgment that most of the decision making problems can be verbally described. Among the VDA framework, the first methods proposed, and also the most known, were ORCLASS, PACOM, and ZAPROS [1] and their characteristics and applications can be found in [4-9].

According to [2], methods on MCDA are usually not capable of working with verbal information of preferences, and procedures are applied in order to convert these preferences to a numerical form. However, there might be losses of 


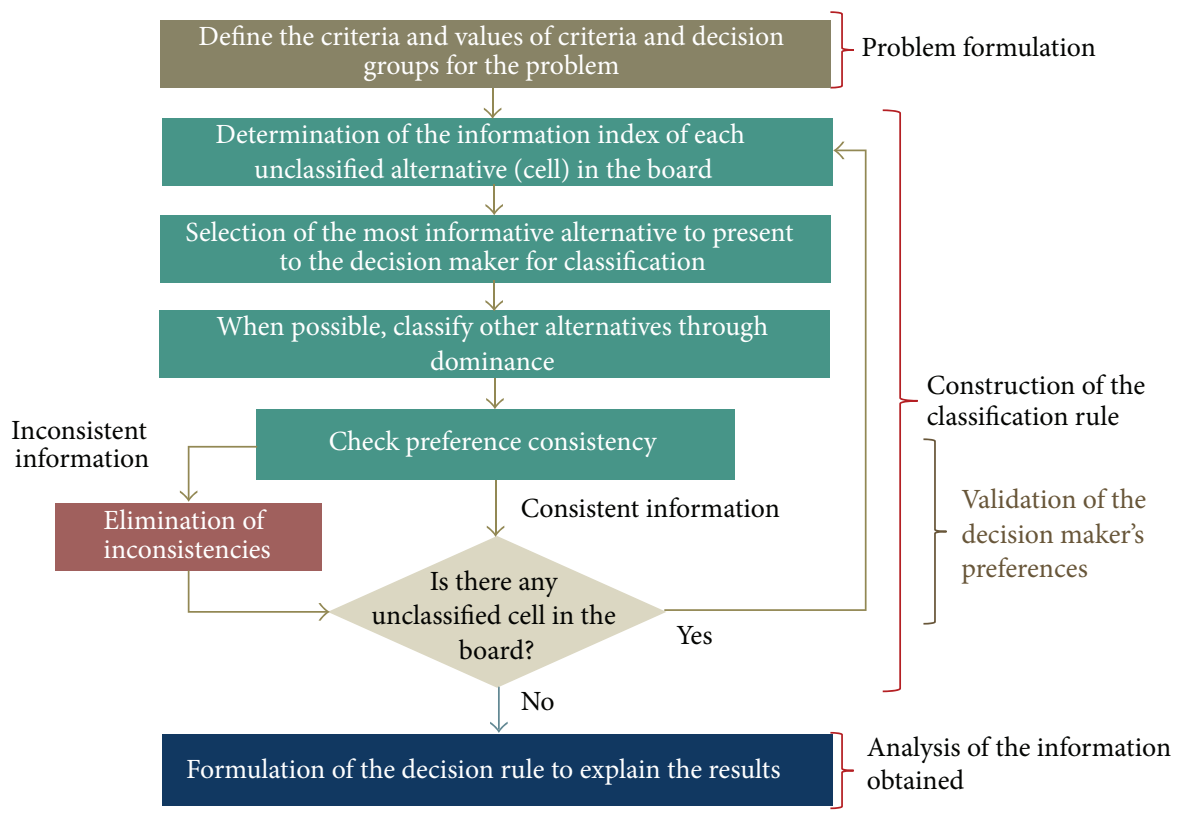

FIGURE 1: Procedure to apply the ORCLASS method.

information when trying to assign numerical values to verbal measurements. Thus, procedures that are able to work with verbal information of preferences are more consistent and stable [10]. Thus, this work focuses on the Verbal Decision Analysis methods [1] which are categorized as nonclassical approaches of MCDA, in particular, the ORCLASS method.

Regarding the choice of the multicriteria approaches, an important point is to evaluate the problem, the objects of decision and information available. According to [11], the choice of the method should be the result of an evaluation of the chosen parameters, the type and accuracy of the data, the way of thinking of the decision maker, and his/her knowledge of the problem. It is noteworthy that the direct consequence of the choice between various methods is that the results can be uneven and even contradictory. Even as, it does not complicate the evaluation, since the differences are more related to diversity that results contradictions and there are some criteria to validate the chosen method [11].

In the issue in question, the application of method ORCLASS came to acceptance by the decision maker, which meant that the issues that were being presented to the decision maker made sense to him, and he had confidence in answering them. Beyond this point, extolled the need to assess the acceptance of the data, its properties used by the method, and the results supported the decision process. Secondary issues such as the existence of tools were also observed, as those would allow greater integration with the problem addressed. Still in the view of the authors, they agree with [12], which stressed that the methodology for multicriteria decision has several methods that can be applied in various problems.

This way, objecting to solve complex problems and provide better utilization of ORCLASS, the new tool OrclassWeb was developed to reproduce the mentioned methodology automatically.

\section{ORCLASS Method-Overview and Structure}

The ORCLASS method (Ordinal Classification) is focused in classifying multicriteria alternatives, which are the options for solving the problem and can be characterized according to the criteria provided. The method differs from other Verbal Decision Analysis methods (such as ZAPROS and PACOM) because it does not aim at ordering alternatives but at classifying all possible multicriteria alternatives, which are given by the Cartesian product of the criteria values defined for the problem. The method aims at categorizing the alternatives into a small number of decision classes or groups, which are preordered according to the decision maker's preferences [1].

2.1. ORCLASS Structure. In fact, many different methods for solving multicriteria classification problems are widely known. The ordinary classification method ORCLASS was one of the first methods designed to solve these kinds of problems. Moreover, recent methods appeared, such as SAC and CYCLE [13]. ORCLASS methodology aims at classifying the alternatives in a given set: the decision maker needs these alternatives to be categorized into a small number of decision classes or groups, usually two. The first group covers the most preferable alternatives, and the less preferable alternatives belong to the second one. However, more than two classes might be defined for a problem. According to [8], Figure 1 presents a flowchart with steps to apply the method ORCLASS. In accordance with the scheme described 
in Figure 1, the application of the method can be divided into three stages: Problem Formulation, Structuring of the Classification Rule, and Analysis of the Information Obtained.

2.2. Formal Statement of the Problem. The methodology follows the same problem formulation proposed in [1].

Given. (1) $K=1,2, \ldots, N$, representing a set of $N$ criteria.

(2) $n_{q}$ represents the number of possible values on the scale of $q$ th criterion $(q \in K)$; for the ill-structured problems, as in this case, usually $n_{q} \leq 4$.

(3) $X_{q}=\left\{x_{i q}\right\}$ represents a set of values to the $q$ th criterion, which is this criterion scale; $\left|X_{q}\right|=n_{q}(q \in K)$; the values of the scale are ranked from best to worst, and this order does not depend on the values of other scales.

(4) $Y=X_{1} * X_{2} * \cdots * X_{n}$ represents a set of vectors $y_{i}$ (every possible alternative: hypothetical alternatives + real alternatives), such that $y_{i}=\left(y_{i 1} ; y_{i 2} ; \ldots ; y_{i Q}\right)$, and $y_{i} \in$ $Y, y_{i q} \in X_{q}$, and $Q=|Y|$, such that $|Y|=\prod_{q=1}^{Q} n_{q}$.

(5) $A=\left\{a_{i}\right\} \in Y, i=1,2, \ldots, t$, such that the set of $t$ vectors represents the description of the real alternatives.

(6) $L$ is number of ordered decision classes.

Required. The multicriteria alternatives classification based on the decision maker's preferences (judgment) to build a reflection.

2.3. Method's Application. At first, in the problem's formulation stage, the set of criteria, criteria values, and the decision groups are defined. The criteria values must be sorted in ascending order of preference (from most preferable to least).

Then, the construction of the classification rule will be carried out based on the decision maker's preferences. In order to present the method's application in a comprehensive way, we use the same concepts presented in [1], on which a classification task is presented as a set of boards.

Each cell is composed by a combination of values for each criterion defined to the problem, which represents a possible alternative to the problem. Let us consider a problem containing the criteria $A, B$, and $C$, the criteria values: $A=\{A 1$, $\mathrm{A} 2, \mathrm{~A} 3\}, \mathrm{B}=\{\mathrm{B} 1, \mathrm{~B} 2, \mathrm{~B} 3\}, \mathrm{C}=\{\mathrm{C} 1, \mathrm{C} 2, \mathrm{C} 3\}$, and two decision classes (I and II), so that, according to the decision maker, the first class is preferable to the second one. For the given problem, we can say the following.

(i) An alternative composed by the best characteristics ([A1, B1, C1]) will always belong to Class I.

(ii) An alternative composed by the worst characteristics ([A3, B3, C3]) will always belong to Class II.

As defined in [1] if a possible alternative composed by the criterion values [A1, B1, C3] is presented to the decision maker for judgment, for example, and supposing that the decision maker classifies the alternative in the first group, then we can infer that a better alternative as [A1, B1, C2] certainly belongs to the first group, since the last one is naturally more preferable than the previous. This response would fill two cells of the classification board. However, if the decision maker judges that the alternative from the example $[\mathrm{A} 1, \mathrm{~B} 1, \mathrm{C} 3]$ is classified into the second group, then the worst
TABLE 1: Classification boards composed by the amount of generated information.

(a) $\mathrm{Cl}$

\begin{tabular}{lccc}
\hline & B1 & B2 & B3 \\
\hline A1 & I & $1+17$ & $2+8$ \\
A2 & $1+17$ & $3+11$ & $5+5$ \\
A3 & $2+8$ & $5+5$ & $8+2$ \\
\hline
\end{tabular}

(b) $\mathrm{C} 2$

\begin{tabular}{lccc}
\hline & B1 & B2 & B3 \\
\hline A1 & $1+17$ & $3+11$ & $5+5$ \\
A2 & $3+11$ & $7+7$ & $11+3$ \\
A3 & $5+5$ & $11+3$ & $17+1$ \\
\hline
\end{tabular}

(c) $\mathrm{C} 3$

\begin{tabular}{lccc}
\hline & B1 & B2 & B3 \\
\hline A1 & $2+8$ & $5+5$ & $8+2$ \\
A2 & $5+5$ & $11+3$ & $17+1$ \\
A3 & $8+2$ & $17+1$ & II \\
\hline
\end{tabular}

alternatives also will be classified as group 2. This way, the alternatives [A1, B2, C3], [A1, B3, C3], [A2, B1, C3], [A2, B2, C3], [A2, B3, C3], [A3, B1, C3], and [A3, B2, C3] will belong to the second decision group, since they are naturally less preferable than the previous. This response would fill eight cells of the classification board.

The additional cells are classified based on dominance relations when the decision maker classifies a determined cell of the table. Moreover, Table 1 presents the classification board for illustrating and better visualization of the amount of cells that could be additionally classified based on the decision maker's preferences. The left number on each cell represents the amount of cells that would be additionally classified in case that cell belongs to class I, and the right number represents the number of additionally classified cells in case that cell belongs to class II. These numbers represent the informative index of each cell.

Only the combinations that, when classified, will reflect this information to a subset of the possible alternatives will be presented to the decision maker for classification, and only the cell with the most informative index will be considered. The one that presents the smallest difference between the indexes and the maximum sum of these indexes will give the most informative cell. In case of a tie between the alternatives, one can randomly choose one of them. In conclusion, the most informative cell is given by [A2, B2, C2] (which enables seven new classifications regardless of which class it belongs to), and it is the best option to be presented to the decision maker for classification. Supposing that cell [A2, B2, C2] was classified as class I, Table 2 presents the new informative indexes after this classification.

In addition, when the problem contains only two classes, it is necessary to check the consistency of the decision maker's answers. On the detection of inconsistent information, these should be presented to the decision maker for analysis and correction. The checking can be made by asking the decision 
TABLE 2: Classification boards composed by the amount of generated information once the first classification is made.

(a) $\mathrm{Cl}$

\begin{tabular}{lccc}
\hline & B1 & B2 & B3 \\
\hline A1 & I & I & $1+8$ \\
A2 & I & I & $2+5$ \\
A3 & $1+8$ & $2+5$ & $5+2$ \\
\hline
\end{tabular}

(b) $\mathrm{C} 2$

\begin{tabular}{lccc}
\hline & B1 & B2 & B3 \\
\hline A1 & I & I & $2+5$ \\
A2 & I & I & $4+3$ \\
A3 & $2+5$ & $4+3$ & $10+1$ \\
\hline
\end{tabular}

(c) $\mathrm{C} 3$

\begin{tabular}{lccc}
\hline & B1 & B2 & B3 \\
\hline A1 & $1+8$ & $2+5$ & $5+2$ \\
A2 & $2+5$ & $4+3$ & $10+1$ \\
A3 & $5+2$ & $10+1$ & II \\
\hline
\end{tabular}

maker to classify an already classified cell on the board. The classification should respect the dominance relations, so that an alternative with better criteria values should not be classified into a group that is considered not preferable from the decision maker's point of view, once, at the same time, the group that is considered better contains alternatives with worse criteria values. In problems with more than two classes, the check of consistency is automatically performed within the classification task. More information about the ORCLASS method is available in [1].

2.4. Tools Based on Verbal Decision Analysis. There are a few tools for automating Verbal Decision Analysis (VDA) methodologies. For methods that aim at the ordering of alternatives, for example, we can cite the one described in detail by Dimitriadi and Larichev [14], which illustrates an implementation of a different version of ZAPROS-III in Visual $\mathrm{C}++6.0$, not applying Formal Index of Quality (FIQ) for alternatives comparing process. Still for ordering of alternatives purposes, we have UniComBOS [15], which applies its own method to determine the order of given alternatives, implementing a new procedure for comparison and choice of multicriteria alternatives. As for the ZAPROSIII method, Tamanini [8] implements a tool focused on treatment of incomparability. The work reduces problems of incomparability and improves the method ZAPROS-III $[16,17]$, generating the new approach methodology ZAPROS III- $i$.

In the alternatives classification scenario, a tool for the method SAC (or CLARA) is presented in [18]. Different from the ORCLASS method, it aims at classifying the alternatives that are relevant to the problem being approached. It also differs from the ORCLASS one on the form that it calculates the information index of the alternatives: it considers a variation measurement of the number of indirectly classified alternatives. Some recommendations for the variance values are presented in [2]. When the variance value is 0 , the information index of the alternatives is calculated on the same way of the ORCLASS method.

Thus, the motivation of creating a new tool to implement the ORCLASS method is justified by the fact that, for research purposes, the authors wanted to have a final classification rule that would apply to any alternative that is described based on the established criteria values. This way, the multicriteria models would be more generic and the alternatives could be changed without impacting on the final results.

\section{A Tool for the ORCLASS Method-The OrclassWeb Tool}

In order to facilitate the decision making process using ORCLASS and perform it consistently, observing its rules and aiming at making it accessible, a tool developed in platform Java Web that applies the methodology is presented [19]. The tool was built in a web environment in Platform Java 1.6 using JSF 2 and runs in server Tomcat 6. OrclassWeb tool was proposed to automate the comparison process of alternatives and to provide the decision maker a concrete result for the problem, according to ORCLASS definition. OrclassWeb was developed divided into four stages:

(1) criteria and criteria values definition,

(2) alternatives definition,

(3) construction of the classification rule,

(4) presentation of results obtained.

The manual application of the system ORCLASS is usually made with a maximum of three criteria and three criteria values for each one, because the complexity of the application increases exponentially with the growth of these numbers. This way, the main advantage of OrclassWeb is that the tool processes the complexity of the application, which means that the user can apply ORCLASS with a greater number of criteria and criteria values. OrclassWeb was developed adapting the rules to identify the most informative cell, after applying the rules defined by [1]:

After the identification of the most informative index according to the rules, the tool verifies among all the others alternatives, which one presents the highest values for both indexes; being this the most informative cell of the board.

In conclusion, the adaptation was necessary to increase the method's comparison capacity, without giving away the adherence to the ORCLASS method. The tool's interfaces and its features are presented in Sections 3.1 and 3.2, which describe the stages of the ORCLASS application. For research purposes, the tool can be reached at http://www2.unifor.br/OrclassWeb.

3.1. OrclassWeb: Interfaces and Availability. First of all, at the beginning of the application it is necessary to define the criteria and criteria values associated that the alternatives will be compared against, presented in the problem. Figure 2 


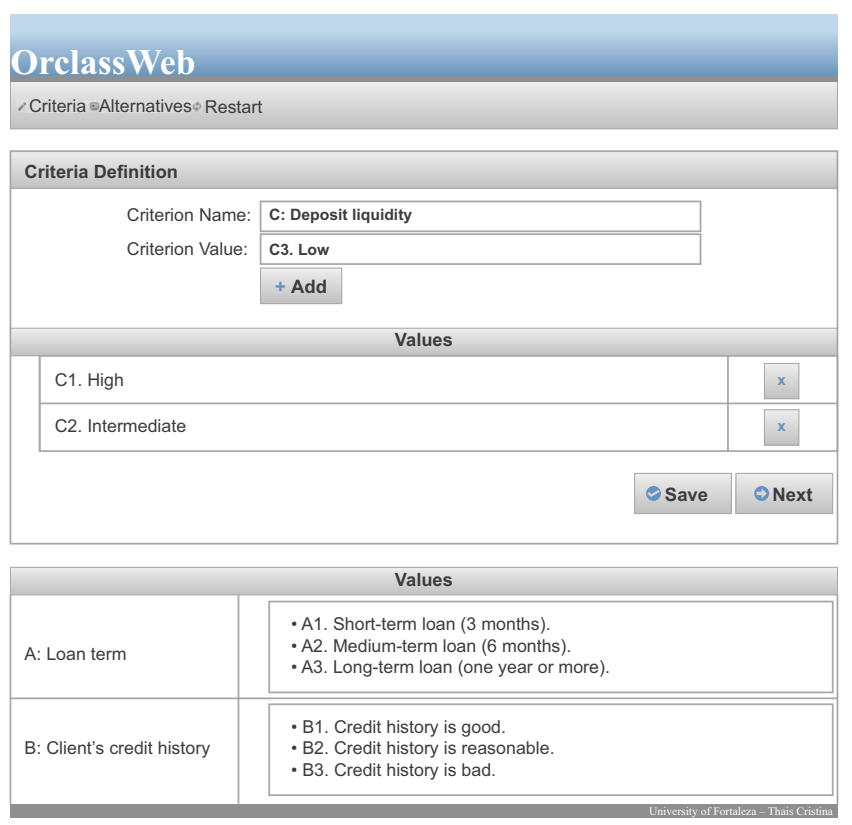

Figure 2: Criteria definition interface: who is worth the loan?

presents the tool's interface for defining criteria and criteria values. This is the stage named "Problem Formulation" from the procedure. The second stage of OrclassWeb is the definition of the alternatives. This is the step which the decision maker states the alternatives and characterize them according to the criteria values. Figure 3 presents the tool's interface for defining alternatives. This is the phase named "Construction of the Classification Rule" of the procedure.

The next step of OrclassWeb is the elicitation of preferences stage. The interface for elicitation of preferences presents questions that can be easily answered by the decision maker in order to obtain the scale of preferences. According to the decision maker's responses, OrclassWeb initiates the division of alternatives into the classes/groups. Figure 4 presents the tool's interface for capturing preferences. The current step also belongs to "Construction of the Classification Rule" phase from the procedure. As the last step for applying the method, at the end of the comparison process OrclassWeb presents an interface composed by the groups and the related alternatives preferred for each one in order to allow a complete and more detailed analysis of the results obtained. Table 3 presents the tool's interface for the analysis results. This interface provides the visualization of "Analysis of Information Obtained" stage from procedure.

OrclassWeb is a tool developed for web, not a Desktop application. It means that the system can be accessed everywhere, via Internet, through the website.

3.2. OrclassWeb: Reliability and Exemplification of Use. Functional tests were elaborated and executed aiming at testing the OrclassWeb and stating it as a reliable and effective tool. The tests were done by applying ORCLASS system manually and were also applied through the tool. The results are compared in order to identify that OrclassWeb attends to all

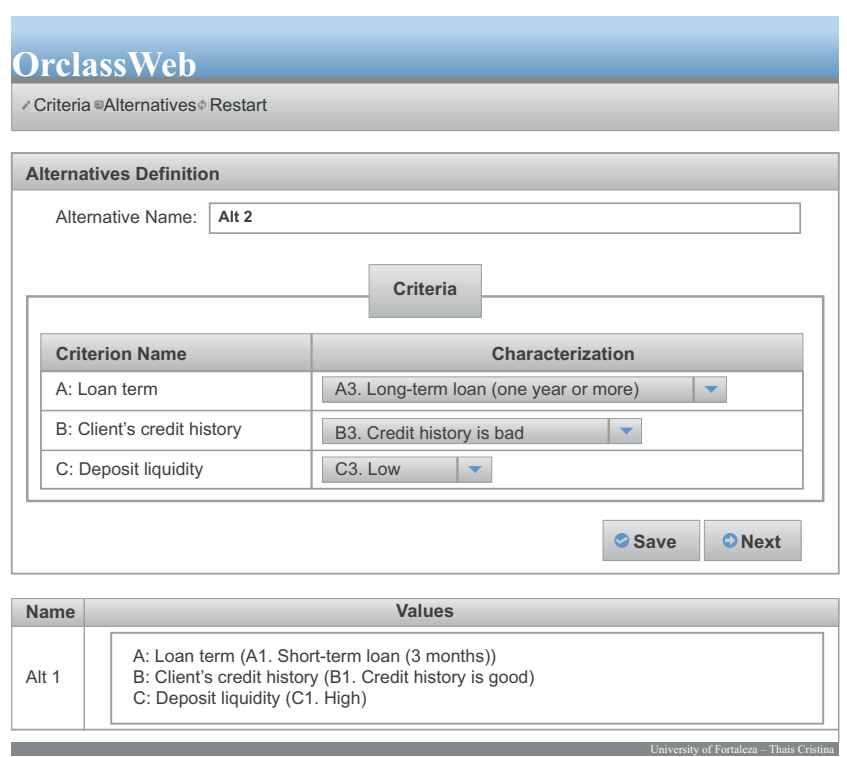

FIGURE 3: Alternatives definition interface: who is worth the loan?

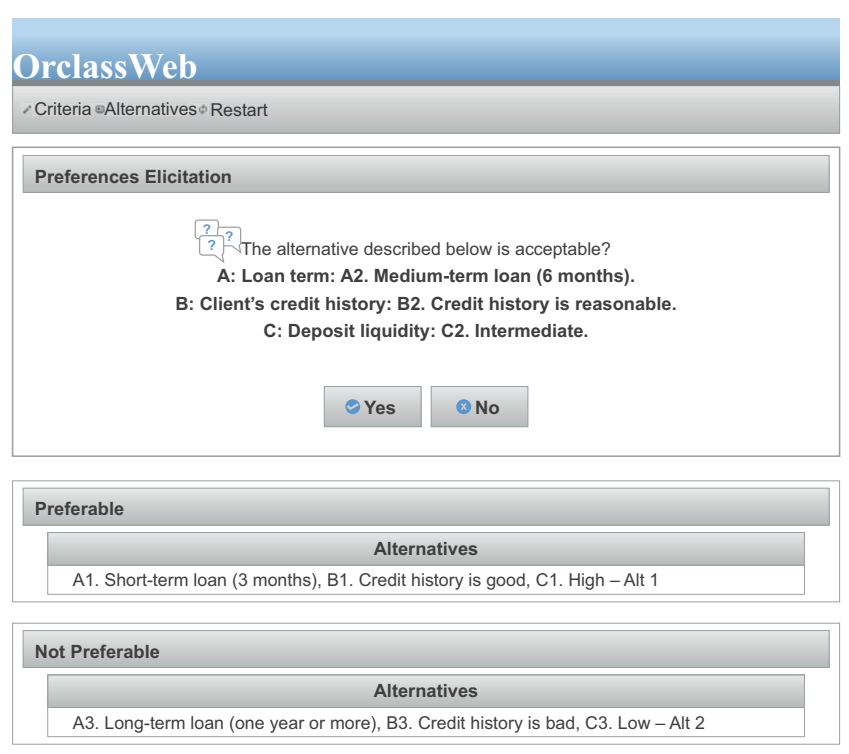

FIGURE 4: Preferences elicitation interface: who is worth the loan?

the requirements imposed by the methodology. As example of applications that used the tool to aid in the method's application, we have the applications presented in [20,21].

For the following section, we show the tool's application to the problem presented in [1]. The application occurs exactly as defined in the procedure for the classification methodology ORCLASS. After that, we provide an original multicriteria problem to exemplify the use of the tool. The results obtained with OrclassWeb will be compared to the ones obtained through the manual application of the method to problems previously published.

3.2.1. Application of the Tool to the Multicriteria Problem: Who Is Worth the Loan? The first problem stated used to 
TABLE 3: Results presentation interface: who is worth the loan?

Final result

Preferable

A1. Short-term loan (3 months). B1. Credit history is good. C1. High-Alt 1

A2. Medium-term loan (6 months). B2. Credit history is reasonable. C2. Intermediate.

A1. Short-term loan (3 months). B2. Credit history is reasonable. C2. Intermediate.

A2. Medium-term loan (6 months). B1. Credit history is good. C2. Intermediate.

A2. Medium-term loan (6 months). B2. Credit history is reasonable. C1. High.

A1. Short-term loan (3 months). B1. Credit history is good. C2. Intermediate.

A1. Short-term loan (3 months). B2. Credit history is reasonable. C1. High.

A2. Medium-term loan (6 months). B1. Credit history is good. C1. High.

A3. Long-term loan (one year or more). B1. Credit history is good. C2. Intermediate.

A3. Long-term loan (one year or more). B1. Credit history is good. C1. High.

A1. Short-term loan (3 months). B3. Credit history is bad. C1. High.

Not preferable

A3. Long-term loan (one year or more). B3. Credit history is bad. C3. Low-Alt 2

A3. Long-term loan (one year or more). B2. Credit history is reasonable. C2. Intermediate.

A3. Long-term loan (one year or more). B3. Credit history is bad. C2. Intermediate.

A3. Long-term loan (one year or more). B2. Credit history is reasonable. C3. Low.

A1. Short-term loan (3 months). B3. Credit history is bad. C3. Low.

A2. Medium-term loan (6 months). B3. Credit history is bad. C3. Low.

A2. Medium-term loan (6 months). B3. Credit history is bad. C1. High.

A3. Long-term loan (one year or more). B3. Credit history is bad. C1. High.

A2. Medium-term loan (6 months). B3. Credit history is bad. C2. Intermediate.

A2. Medium-term loan (6 months). B1. Credit history is good. C3. Low.

A3. Long-term loan (one year or more). B1. Credit history is good. C3. Low.

A2. Medium-term loan (6 months). B2. Credit history is reasonable. C3. Low.

A3. Long-term loan (one year or more). B2. Credit history is reasonable. C1. High.

A1. Short-term loan (3 months). B2. Credit history is reasonable. C3. Low.

A1. Short-term loan (3 months). B1. Credit history is good. C3. Low.

A1. Short-term loan (3 months). B3. Credit history is bad. C2. Intermediate.

exemplify the tool's use is the evaluation of loan applications defined in [1]. This problem was used originally by [1] to explain ORCLASS method application procedure. The situation consists in a group of businesses submitting a loan application to a commercial bank. In what cases the loan should be rejected or accepted? The bank is the decision maker and provides a policy for granting loans. The decision maker is the person to whom the decision will be taken, and the structuring of the classification rule in the OrclassWeb was done based on the rule already defined on the original problem resolution. Table 4 presents the list of criteria and criteria values, which will be base to apply the methodology. The criteria values are described from the naturally most preferable to the least preferable one.

Criteria and criteria values are required by the tool. The criteria values need to be entered in order, according to their preference degrees, through the "Criteria Definition Interface," shown in Figure 2.

The alternatives for the application will be hypothetical situations, since the problem definition in [1] does not
TABLE 4: Criteria and associated values: who is worth the loan?

\begin{tabular}{ll}
\hline Criteria & Criteria values \\
\hline \multirow{3}{*}{ A: Loan term } & A1. Short-term loan (3 months). \\
& A2. Medium-term loan (6 months). \\
& A3. Long-term loan (one year or more). \\
\hline \multirow{3}{*}{ B: Client's credit history } & B1. Credit history is good. \\
& $\begin{array}{l}\text { B2. Credit history is reasonable. } \\
\text { B3. Credit history is bad. }\end{array}$ \\
\hline & C1. High. \\
C: Deposit liquidity & C2. Intermediate. \\
& C3. Low.
\end{tabular}

describe the alternatives. Table 5 presents the list of alternatives for this case.

Alternatives are also required objects by the tool, and they should have a name and be characterized based on criteria values. The "Alternatives Definition Interface" of OrclassWeb can be visualized in Figure 3. 
TABLE 5: Identification of alternatives board: who is worth the loan?

\begin{tabular}{ll}
\hline ID & Alternatives \\
\hline Alt 1 & Represents the best alternative \\
Alt 2 & Represents the worst alternative \\
\hline
\end{tabular}

TABLE 6: Result matrix: who is worth the loan?

(a) $\mathrm{Cl}$

\begin{tabular}{lccc}
\hline & B1 & B2 & B3 \\
\hline A1 & I & I & I \\
A2 & I & I & II \\
A3 & I & II & II \\
\hline
\end{tabular}

(b) $\mathrm{C} 2$

\begin{tabular}{lccc}
\hline & B1 & B2 & B3 \\
\hline A1 & I & I & II \\
A2 & I & I & II \\
A3 & I & II & II \\
\hline
\end{tabular}

(c) $\mathrm{C} 3$

\begin{tabular}{llll}
\hline & B1 & B2 & B3 \\
\hline A1 & II & II & II \\
A2 & II & II & II \\
A3 & II & II & II \\
\hline
\end{tabular}

After all the criteria and alternatives are defined, the user's preferences will be accessed through the "Preferences Elicitation Interface," as presented in Figure 4. Note that the user is not required to have a deep knowledge of the method to be able to use the tool. The information indexes are used at this part of the process, following the rules presented in [1].

The tool conducts the entire process of elicitation of preferences. The process can be applied manually using a board that will be filled according to the decision maker preferences. For both cases, the logic involved is the same: the application of ORCLASS is made calculating the most informative index, which will provide the next question to be posed to the decision maker. Afterwards, the most informative cell from the board is exactly the next question presented to the user of OrclassWeb. Comparing both application techniques, we notice that the same is obtained and can be visualized in Tables 3 and 6.

The use of the tool facilitates the process since the user does not need to calculate the information indexes manually. For problems having three or more criteria, this task can be very stressful and time consuming. Also, no formal knowledge about the method's application is required once using the tool, since one just needs to follow the steps and answer the required questions.

\subsubsection{Application of the Tool to the Multicriteria Problem:} Selecting Practices of Framework SCRUM. Considering that agile methodologies, in particular the Framework SCRUM $[10,21-24]$, are more and more common in development software companies and noticing that the mentioned companies cannot always apply every approach of the framework, the

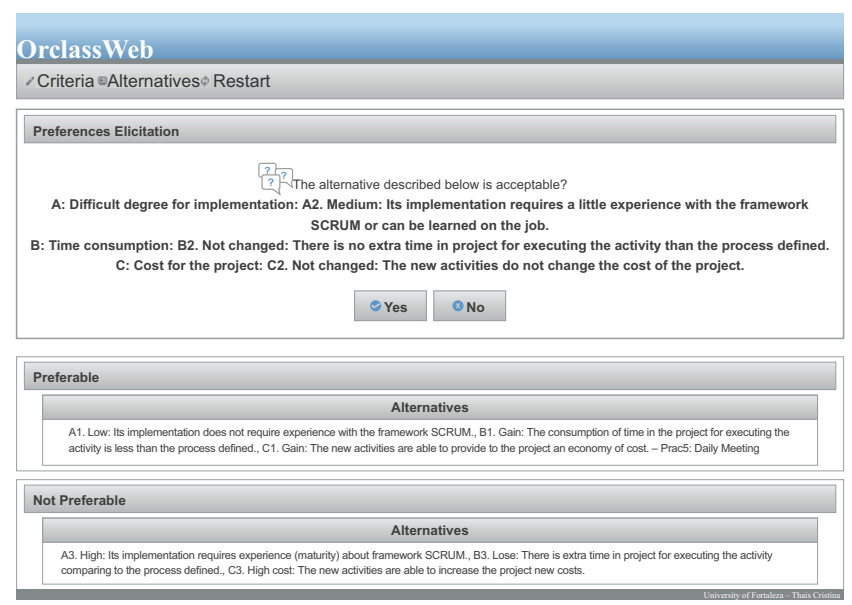

FIgURE 5: Preferences elicitation interface: selecting practices of Framework SCRUM.

research applies the OrclassWeb tool aiming at selecting the approaches to be applied in the company, considering the elicitation of preferences of a decision maker. The application consists in the classification of SCRUM approaches in groups or classes. The first group was chosen to support the practices of Framework SCRUM which should be selected after the application of ORCLASS, to be implanted by the organizations, and the second class will support the set of approaches that should not be implanted by the organization which desire to implement part of SCRUM. Table 7 presents the list of criteria and criteria values, which will be used to evaluate the set of alternatives. These were required to be entered into the tool according to its preference degrees (following the order shown in the table).

The alternatives for the application will be SCRUM approaches and they are listed in Table 8. The alternatives were entered into the tool through the "Alternatives Definition Interface."

The process of elicitation of preferences is conducted by OrclassWeb, tool in parallel to the application made manually, through "Preferences Elicitation Interface," as presented in Figure 5.

The tool conducts the entire process of elicitation of preferences, and, manually, the process can be applied using a table, which will be filled according to the decision maker preferences. Manually, the application of ORCLASS is made calculating the most informative index, which will provide the next question to be posed to the decision maker. Afterwards, the most informative cell from the board is exactly the next question presented to the user of OrclassWeb. The conclusion of elicitation of preferences is reached in both tools: OrclassWeb and manually. The result is the same and can be visualized in Tables 9 and 10 .

Comparing the result obtained in both situations, the first and the second group are composed by the SCRUM approaches as described below.

(i) Class 1 is composed by the following SCRUM approaches: 
TABLE 7: Criteria and associated values: selecting practices of Framework SCRUM.

\begin{tabular}{ll}
\hline Criteria & Criteria values \\
$\begin{array}{l}\text { A: Difficult degree for } \\
\text { implementation }\end{array}$ & $\begin{array}{l}\text { A1. Low: its implementation does not require experience with the framework SCRUM. } \\
\text { A2. Medium: its implementation requires a little experience with the framework SCRUM or can be learned on } \\
\text { the job. } \\
\text { A3. High: its implementation requires experience (maturity) about framework SCRUM. }\end{array}$ \\
\hline B: Time consumption & $\begin{array}{l}\text { B2. Noin: the consumption of time in the project for executing the activity is less than the process defined. } \\
\text { B3. Lose: there is extra time in project for executing the activity comparing to the process defined. }\end{array}$ \\
\hline & C1. Gain: the new activities are able to provide to the project an economy of cost. \\
C: Cost for the project & $\begin{array}{l}\text { C2. Not changed: the new activities do not change the cost of the project. } \\
\text { C3. High cost: the new activities are able to increase the project new costs. }\end{array}$
\end{tabular}

TABLE 8: Identification of alternatives board: selecting practices of Framework SCRUM.

\begin{tabular}{|c|c|}
\hline ID & Alternatives \\
\hline Pracl & Sprints (or iterations) with 1 to 4 weeks \\
\hline Prac2 & $\begin{array}{l}\text { A product backlog and a sprint backlog creation and } \\
\text { prioritization }\end{array}$ \\
\hline Prac3 & Planning meeting—part 1 \\
\hline Prac4 & Planning meeting-part 2 \\
\hline Prac5 & Daily meeting \\
\hline Prac6 & Burn down chart and visible activities board \\
\hline Prac7 & Sprint review \\
\hline Prac8 & Sprint retrospective \\
\hline Prac9 & Release planning \\
\hline
\end{tabular}

TABLE 9: Result matrix: selecting practices of Framework SCRUM.

(a) $\mathrm{Cl}$

\begin{tabular}{lccc}
\hline & B1 & B2 & B3 \\
\hline A1 & I & I & I \\
A2 & I & I & I \\
A3 & II & II & II \\
\hline
\end{tabular}

(b) $\mathrm{C} 2$

\begin{tabular}{lccc}
\hline & B1 & B2 & B3 \\
\hline A1 & I & I & I \\
A2 & I & I & II \\
A3 & II & II & II \\
\hline
\end{tabular}

(c) $\mathrm{C} 3$

\begin{tabular}{lccc}
\hline & B1 & B2 & B3 \\
\hline A1 & I & II & II \\
A2 & II & II & II \\
A3 & II & II & II \\
\hline
\end{tabular}

(a) Pracl: sprints (or iterations) with 1 to 4 weeks,

(b) Prac5: daily meeting,

(c) Prac6: burn down chart and visible activities board, (d) Prac7: sprint review,

(e) Prac8: sprint retrospective,

(f) Prac9: release planning.

(ii) Class 2 is composed by SCRUM approaches:

(a) Prac2: a product backlog and a sprint backlog creation and prioritization,

(b) Prac3: planning meeting-part 1,

(c) Prac4: planning meeting-part 2.

\section{Conclusions and Future Works}

The decision may be defined as a result of a process of choice, given an identified problem or when the decision maker faces an opportunity of creation, optimization, or improvement in an environment. Several factors, which might be measurable, usually influence on the decision making process. It means that the decision is made according to the decision maker preferences.

Observing that the decision maker is capable of manifesting his preferences and interests, it is sufficient to solve simple problems. If the decision maker needs to solve complex problems covering many alternatives and much information that cannot be measured and easily compared, there are some methodologies to be applied in order to support the decision making process.

The main contribution described in this work was OrclassWeb tool. The software was developed and examples of the tool usage and functioning were provided. The tool is available for anyone through a website for research. In conclusion, the developed tool OrclassWeb is proved as truly reliable and strong enough to support the methodology of Verbal Decision Analysis and perform it consistently. Other applications using the SCRUM where the OrclassWeb was applied can be found in $[20,25,26]$.

As future works, other tools can be developed in order to reproduce other DSS methodologies not provided automatically yet. Furthermore, more research can be done applying the OrclassWeb tool with more than three criteria and criteria values in known published problems. There is also the intention to refine the software in order to provide more 
TABLE 10: Results presentation interface: selecting practices of Framework SCRUM.

Final result

Preferable

A1. Low: its implementation does not require experience with the framework SCRUM. B1. Gain: the consumption of time in the project for executing the activity is less than the process defined. Cl. Gain: the new activities are able to provide to the project an economy of cost-Prac5

A2. Medium: its implementation requires a little experience with the framework SCRUM or can be learned on the job. B2. Not changed: there is no extra time in project for executing the activity compared to the process defined. C2. Not changed: the new activities do not change the cost of the project-Prac6

A1. Low: its implementation does not require experience with the framework SCRUM. B2. Not changed: there is no extra time in project for executing the activity compared to the process defined. C2. Not changed: the new activities do not change the cost of the project.

A2. Medium: its implementation requires a little experience with the framework SCRUM or can be learned on the job. B1. Gain: the consumption of time in the project for executing the activity is less than the process defined. C2. Not changed: the new activities do not change the cost of the project.

A2. Medium: its implementation requires a little experience with the framework SCRUM or can be learned on the job. B2. Not changed: there is no extra time in project for executing the activity than the process defined. C1. Gain: the new activities are able to provide to the project an economy of cost.

A1. Low: its implementation does not require experience with the framework SCRUM. B1. Gain: the consumption of time in the project for executing the activity is less than the process defined. C2. Not changed: the new activities do not change the cost of the project.

A1. Low: its implementation does not require experience with the framework SCRUM. B2. Not changed: there is no extra time in project for executing the activity compared to the process defined. C1. Gain: the new activities are able to provide to the project an economy of cost.

A2. Medium: its implementation requires a little experience with the framework SCRUM or can be learned on the job. B1. Gain: the consumption of time in the project for executing the activity is less than the process defined. C1. Gain: the new activities are able to provide to the project an economy of cost-Pracl

A2. Medium: its implementation requires a little experience with the framework SCRUM or can be learned on the job. B3. Lose: there is extra time in project for executing the activity comparing to the process defined. C1. Gain: the new activities are able to provide to the project an economy of cost.

A1. Low: its implementation does not require experience with the framework SCRUM. B3. Lose: there is extra time in project for executing the activity comparing to the process defined. C1. Gain: the new activities are able to provide to the project an economy of cost.

A1. Low: its implementation does not require experience with the framework SCRUM. B1. Gain: the consumption of time in the project for executing the activity is less than the process defined. C3. High cost: the new activities are able to increase the project new costs-Prac8

A1. Low: its implementation does not require experience with the framework SCRUM. B3. Lose: there is extra time in project for executing the activity comparing to the process defined. C2. Not changed: the new activities do not change the cost of the project.

Not preferable

A3. High: its implementation requires experience (maturity) about framework SCRUM. B3. Lose: there is extra time in project for executing the activity comparing to the process defined. C3. High cost: the new activities are able to increase the project new costs-Prac4

A3. High: its implementation requires experience (maturity) about framework SCRUM. B2. Not changed: there is no extra time in project for executing the activity compared to the process defined. C2. Not changed: the new activities do not change the cost of the project.

A3. High: its implementation requires experience (maturity) about framework SCRUM. B3. Lose: there is extra time in project for executing the activity comparing to the process defined. C2. Not changed: the new activities do not change the cost of the project. A3. High: its implementation requires experience (maturity) about framework SCRUM. B2. Not changed: there is no extra time in project for executing the activity compared to the process defined. C3. High cost: the new activities are able to increase the project new costs.

A1. Low: its implementation does not require experience with the framework SCRUM. B3. Lose: there is extra time in project for executing the activity comparing to the process defined. C3. High cost: the new activities are able to increase the project new costs. A2. Medium: its implementation requires a little experience with the framework SCRUM or can be learned on the job. B3. Lose: there is extra time in project for executing the activity comparing to the process defined. C3. High cost: the new activities are able to increase the project new costs. 
TABle 10: Continued.

Final result

A2. Medium: its implementation requires a little experience with the framework SCRUM or can be learned on the job. B1. Gain: the consumption of time in the project for executing the activity is less than the process defined. C3. High cost: the new activities are able to increase the project new costs.

A3. High: its implementation requires experience (maturity) about framework SCRUM. B1. Gain: the consumption of time in the project for executing the activity is less than the process defined. C3. High cost: the new activities are able to increase the project new costs.

A2. Medium: its implementation requires a little experience with the framework SCRUM or can be learned on the job. B2. Not changed: there is no extra time in project for executing the activity compared to the process defined. C3. High cost: the new activities are able to increase the project new costs.

A3. High: its implementation requires experience (maturity) about framework SCRUM. B2. Not changed: there is no extra time in project for executing the activity compared to the process defined. C1. Gain: the new activities are able to provide to the project an economy of cost.

A3. High: its implementation requires experience (maturity) about framework SCRUM. B3. Lose: there is extra time in project for executing the activity comparing to the process defined. Cl. Gain: the new activities are able to provide to the project an economy of cost.

A3. High: its implementation requires experience (maturity) about framework SCRUM. B1. Gain: the consumption of time in the project for executing the activity is less than the process defined. C2. Not changed: the new activities do not change the cost of the project.

A2. Medium: its implementation requires a little experience with the framework SCRUM or can be learned on the job. B3. Lose: there is extra time in project for executing the activity comparing to the process defined. C2. Not changed: the new activities do not change the cost of the project.

A3. High: its implementation requires experience (maturity) about framework SCRUM. B1. Gain: the consumption of time in the project for executing the activity is less than the process defined. C1. Gain: the new activities are able to provide to the project an economy of cost.

A1. Low: its implementation does not require experience with the framework SCRUM. B2. Not changed: there is no extra time in project for executing the activity compared to the process defined. C3. High cost: the new activities are able to increase the project new costs-Prac3

treatment of incorrect insertions, storage of results, visual presentation of results, and much more.

\section{Conflict of Interests}

The authors declare that there is no conflict of interests regarding the publication of this paper.

\section{Acknowledgments}

The second and the third authors are thankful to the National Council for Scientific and Technological Development $(\mathrm{CNPq})$ and the first author is thankful to Foundation of Support to the Scientific and Technological Development of the State of Ceara (FUNCAP) for the support received on this project.

\section{References}

[1] O. I. Larichev and H. M. Moshkovich, Verbal Decision Analysis for Unstructured Problems, Kluwer Academic, Dodrecht, The Netherlands, 1997.

[2] I. Yevseyeva, Solving Classification Problems with Multicriteria Decision Aiding Approaches, Jyväskylä University Printing House, Jyväskyl, Finland, 2007.

[3] A. T. Brasil Filho, P. R. Pinheiro, and A. Coelho, "Towards the early diagnosis of Alzheimer s disease via a multicriteria classification model," in Evolutionary Multi-Criterion Optimization, vol. 5467 of Lecture Notes in Computer Science, pp. 393-406, 2009.

[4] T. C. S. Machado, A. C. Menezes, I. Tamanini, and P. R. Pinheiro, "A hybrid model in the selection of prototypes for educational tools: an applicability in verbal decision analysis," in Proceedings of the IEEE Symposium on Computational Intelligence in Multicriteria Decision-Making (MDCM '11), 2011.

[5] T. C. S. Machado, A. C. Menezes, L. F. R. Pinheiro, I. Tamanini, and P. R. Pinheiro, "Applying verbal decision analysis in selecting prototypes for educational tools," in Proceedings of the IEEE International Conference on Intelligent Computing and Intelligent Systems (ICIS '10), pp. 531-535, Xiamen, China, October 2010.

[6] T. C. S. Machado, P. R. Pinheiro, A. B. Albuquerque, and M. M. L. de Lima, "Applying verbal decision analysis in selecting specific practices of CMMI," in International Conference on Rough Sets and Knowledge Technology (RSKT), vol. 7414 of Lecture Notes in Computer Science, pp. 215-221, Springer, Berlin, Germany, 2012.

[7] T. C. S. Machado, P. R. Pinheiro, and I. Tamanini, "Towards the selection of prototypes for educational tools: a hybrid model in the verbal decision analysis," International Journal of Information Science, Scientific \& Academic Publishing, vol. 2, no. 3, pp. 23-32, 2012.

[8] I. Tamanini, Improving the ZAPROS method considering the incomparability cases [M.S. thesis], Graduate Program in Applied Informatics, University of Fortaleza, 2010.

[9] I. Tamanini, A. K. de Castro, P. R. Pinheiro, and M. C. D. Pinheiro, "Verbal decision analysis applied on the optimization 
of alzheimer's disease diagnosis: a case study based on neuroimaging," Advances in Experimental Medicine and Biology, vol. 696, pp. 555-564, 2011.

[10] K. Schwaber, "Agile Project Management with Scrum," Microsoft, 2004.

[11] L. F. A. M. Gomes, H. Moshkovich, and A. Torres, "Marketing decisions in small businesses: how verbal decision analysis can help," International Journal of Management and Decision Making, vol. 11, no. 1, pp. 19-36, 2010.

[12] V. M. Ozernoy, "Choosing the best multiple criteria decision making method," INFOR, vol. 30, no. 2, pp. 159-171, 1992.

[13] L. Ustinovich and D. Kochin, "Verbal decision analysis methods for determining the efficiency of investments in construction," Foundations of Civil and Environmental Engineering, vol. 5, pp. 35-46, 2004.

[14] G. G. Dimitriadi and O. I. Larichev, "Decision support system and the ZAPROS-III method for ranking the multiattribute alternatives with verbal quality estimates," Automation and Remote Control, vol. 66, no. 8, pp. 1322-1335, 2005.

[15] I. Ashikhmin and E. Furems, "UniComBOS-intelligent decision support system for multi-criteria comparison and choice," Journal of Multi-Criteria Decision Analysis, vol. 13, no. 2-3, pp. 147-157, 2005.

[16] O. I. Larichev, "Ranking multicriteria alternatives: the method ZAPROS III," European Journal of Operational Research, vol. 131, no. 3, pp. 550-558, 2001.

[17] O. Larichev, "Method ZAPROS for multicriteria alternatives ranking and the problem of incomparability," Informatica, vol. 12, no. 1, pp. 89-100, 2001.

[18] O. I. Larichev, A. V. Kortnev, and D. Y. Kochin, "Decision support system for classification of a finite set of multicriteria alternatives," Decision Support Systems, vol. 33, no. 1, pp. 13-21, 2002.

[19] T. C. S. Machado, Towards aided by multicriteria support methods and software development: a hybrid model of verbal decision analysis for selecting approaches of project management [M.S. thesis], Graduate Program in Applied Informatics, University of Fortaleza, 2012.

[20] T. C. S. Machado, P. R. Pinheiro, and I. Tamanini, "Project management aided by verbal decision analysis approaches: a case study for the selection of the best SCRUM practices," International Transactions in Operational Research, 2014.

[21] T. C. S. Machado, P. R. Pinheiro, and I. Tamanini, "Dealing the selection of project management through hybrid model of verbal decision analysis," Procedia Computer Science, vol. 17, pp. 332-339, 2013.

[22] K. Beck, M. Beedle, A. van Bennekum et al., "Manifesto for Agile Software Development," 2001, http://agilemanifesto.org/.

[23] K. Schwaber, SCRUM Guide, SCRUM Alliance, 2009.

[24] J. Sutherland and K. Schwaber, The SCRUM Papers: Nuts, Bolts, and Origins of an Agile Process, 2007.

[25] T. C. S. Machado, P. R. Pinheiro, and H. F. Landim, "A hybrid model in the selection of practices of framework SCRUM: an applicability in verbal decision analysis," in Proceedings of the World Summit on the Knowledge Society (WSKS '11), Mykonos, Greece, 2011.

[26] T. C. S. Machado, P. R. Pinheiro, M. L. De Lima Marcelo, and H. F. Landim, "Towards a verbal decision analysis on the selecting practices of framework SCRUM," Lecture Notes in Computer Science, vol. 7030, pp. 585-594, 2011. 


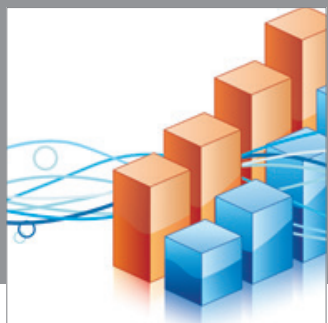

Advances in

Operations Research

mansans

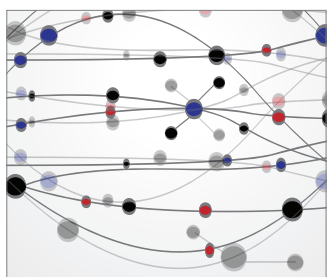

The Scientific World Journal
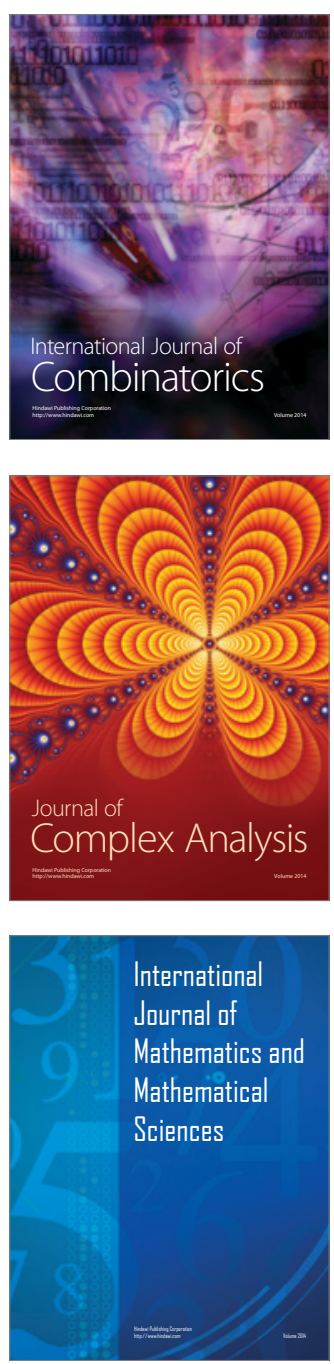
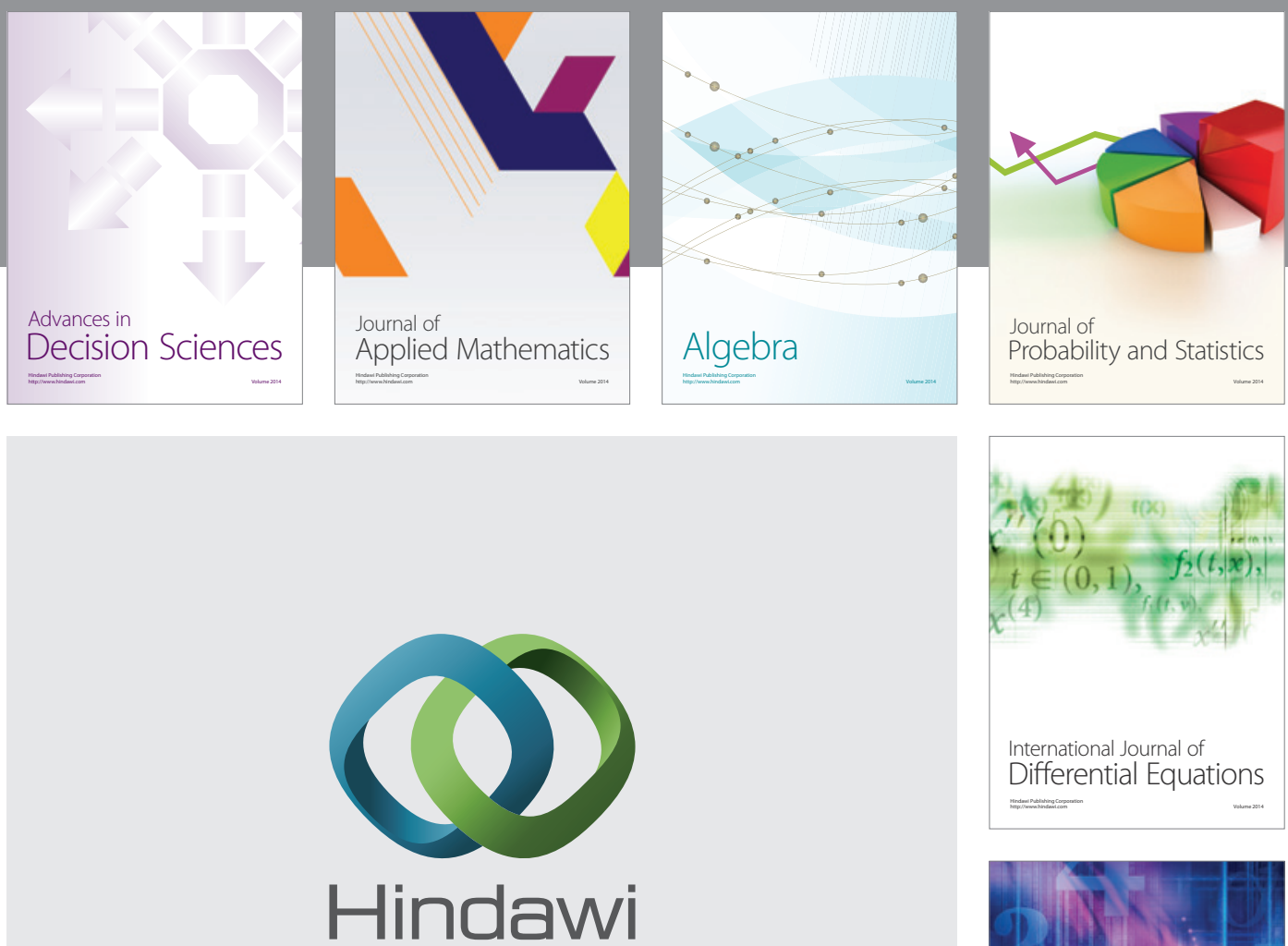

Submit your manuscripts at http://www.hindawi.com
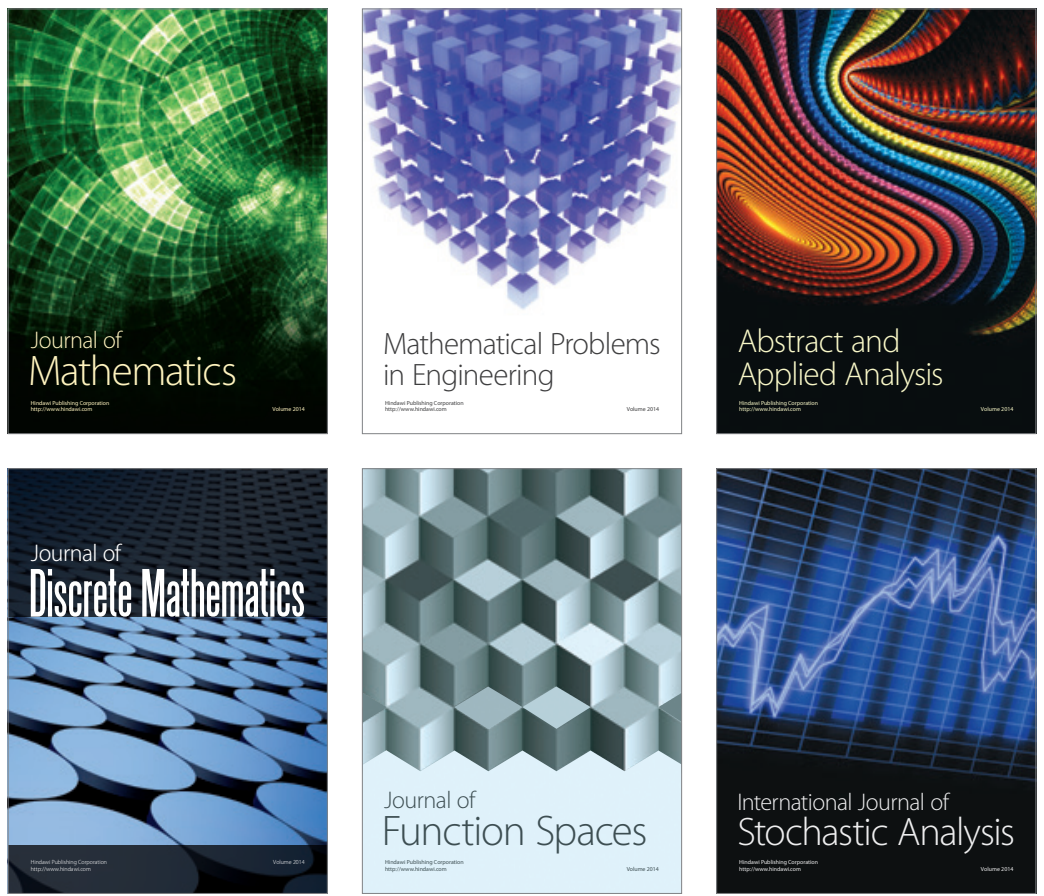

Journal of

Function Spaces

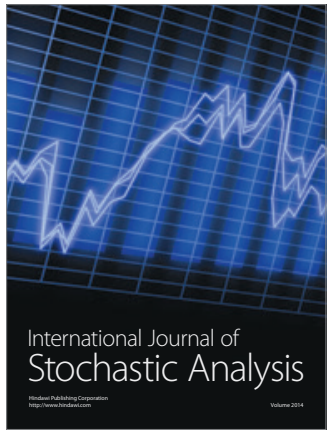

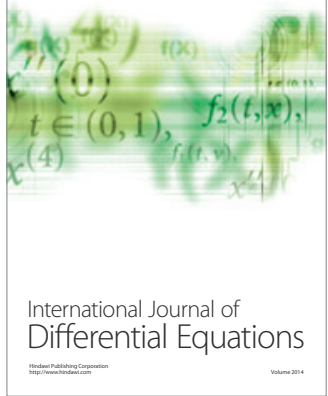
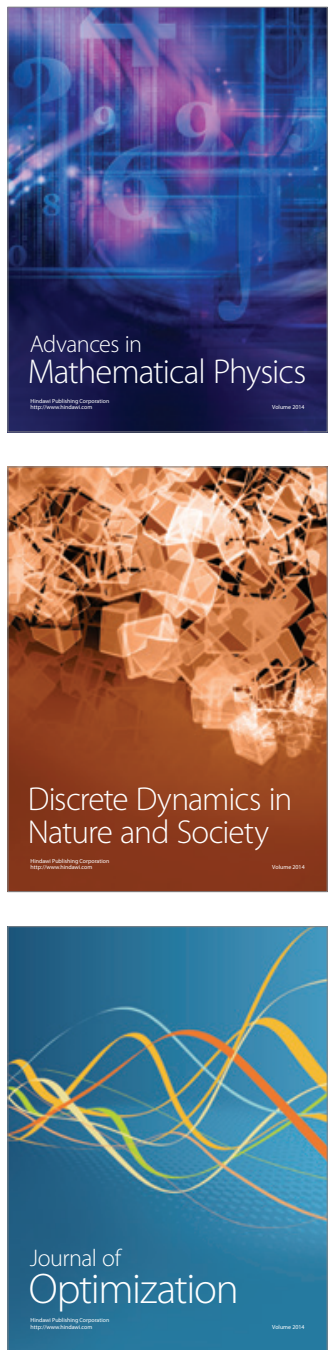